$57^{\text {ème }}$ Congrès de la SFMBCB, 03007 (2011)

DOI:10.1051/sfmbcb/20115703007

(C) Owned by the authors, published by EDP Sciences, 2011

\title{
Intérêt de l'examen clinique en autofluorescence directe dans le dépistage des lésions de la muqueuse buccale
}

\author{
Gaston R, Catros S, Jeandot J, Marteau JM, Fricain JC \\ Pôle Odontologie et Santé buccale, Hôpital Pellegrin, CHU, Bordeaux, France
}

L'autofluorescence directe a été proposée comme outil d'aide au diagnostic pour les cancers de la cavité buccale (Poh 2007, Jayaprakash 2009). Cependant l'aspect des lésions en fluorescence n'est pas spécifique des lésions malignes (Mehotra 2010, Fricain 2010). Aucune étude n'a été réalisée en situation de dépistage lors des soins primaires (Lingen 2008).

L'objectif de cette étude était d'évaluer l'intérêt de l'examen de la muqueuse buccale en autofluorescence directe dans le diagnostic des lésions de la muqueuse buccale sur un échantillon de 147 patients consultant pour des soins dentaires au CHU de Bordeaux. Le matériel utilisé était le VELscope ${ }^{\circledR}$ (LED Dental Inc.). Cet échantillon est composé de 84 hommes et 63 femmes ayant donné leur consentement. Un premier examen visuel standardisé de la muqueuse buccale a été réalisé en lumière blanche. Lors de la même séance, un second examen pour évaluer la fluorescence a été réalisé. Le critère d'évaluation principal était le nombre de lésions détectées par l'examen clinique, en lumière blanche ou en autofluorescence. Le nombre de patients présentant des lésions était de 27 pour les examens en lumière blanche, soit 18,3\% de l'échantillon; 63 lésions ont été observées en tout, soit un nombre moyen de lésions par patient de 2,33 ( $\sigma=1,47)$. L'examen clinique en autofluorescence a mis en évidence 84 lésions chez 34 patients. Ces patients représentaient $23,1 \%$ de l'échantillon, le nombre moyen de lésions par patient était de 2,47 $(\sigma=1,81)$. Une comparaison des moyennes a permis de conclure que le nombre des lésions observées en autofluorescence est significativement plus élevé que celui mesuré en lumière blanche [(DDL $=31$, p-value (bilatérale) $=0.000, \alpha=0,05)]$. Les lésions observées étaient constituées par une malformation vasculaire (2 patients), des lésions inflammatoires d'origine traumatique (17 patients), une mélanose tabagique (5 patients), un lichen plan érosif ( 9 patients), une kératose tabagique (11 patients), une ouranite tabagique ( 2 patients), un papillome (3 patients), une langue géographique ( 5 patients), une fistule et un abcès parodontaux ( 2 patients), une ulcération traumatique (4 patients), une lentigine ( 1 patient), un érythème traumatique (5 patients), une candidose aiguë (7 patients). Les lésions passées inaperçues à l'examen en lumière blanche étaient des lésions inflammatoires traumatiques.

Cette étude semble montrer que l'examen clinique de la muqueuse buccale en autofluorescence détecte plus de lésions que l'examen clinique en lumière blanche. Cependant aucune lésion maligne n'a été détectée ce qui montre qu'en situation de dépistage lors des soins primaires, l'autofluorescence directe ne peut pas être considérée comme un outil diagnostique des lésions à potentiel malin et des cancers. Les données de l'autofluorescence doivent être confrontées aux données de l'examen clinique et de l'anamnèse afin d'être correctement interprétées (Patton 2008).

Correspondance : remgaz@hotmail 